\title{
Models for quantitative charge imaging by atomic force microscopy
}

\author{
Elizabeth A. Boer, ${ }^{\text {a) }}$ L. D. Bell, ${ }^{\text {b) }}$ Mark L. Brongersma, and Harry A. Atwater \\ Thomas J. Watson Laboratory of Applied Physics, California Institute of Technology, Pasadena, \\ California 91125
}

(Received 27 February 2001; accepted for publication 24 June 2001)

\begin{abstract}
Two models are presented for quantitative charge imaging with an atomic-force microscope. The first is appropriate for noncontact mode and the second for intermittent contact (tapping) mode imaging. Different forms for the contact force are used to demonstrate that quantitative charge imaging is possible without precise knowledge of the contact interaction. From the models, estimates of the best charge sensitivity of an unbiased standard atomic-force microscope cantilever are found to be on the order of a few electrons. (C) 2001 American Institute of Physics.
\end{abstract}

[DOI: 10.1063/1.1394896]

\section{INTRODUCTION}

The ability to easily detect charge at the single electron level is becoming increasingly important as interest in charge storage at the few electron scale grows. The atomic-force microscope (AFM) (Ref. 1) is a useful tool to detect small numbers of charges ${ }^{2}$ as well as to inject charge into a localized region. ${ }^{3-9}$ However, quantitative measurement of electrostatic charge has never been straightforward because of the complex factors involved, such as tip shape, tip-sample distances, contamination or oxidation of tip and sample, and the exact nature of the tip-sample interaction. Models, varying in complexity from a parallel-plate capacitor to finiteelement calculations, are always needed to interpret results quantitatively.

The first uses of the AFM to detect electrostatic forces modeled the system as a parallel-plate capacitor ${ }^{10}$ or considered the tip to be a point object. ${ }^{11}$ Since then, several different methods have been proposed for calculating the electrostatic force, ranging from simple analytical calculations ${ }^{12,13}$ and perturbative approaches ${ }^{14}$ to more complex numerical simulations. ${ }^{15-20}$ Much work has been done in modeling of the tip-sample interaction in atomic-force microscopy. ${ }^{21-29}$ In this article, we combine both electrostatic and AFM modeling to interpret AFM images of charge. We describe two models for quantitative charge imaging, one appropriate for noncontact mode imaging, the second for intermittent contact (tapping) mode imaging.

\section{EXPERIMENTS}

The models described in the next sections have been applied to two experimental situations, details of which may be found elsewhere. Briefly, the noncontact mode experiments ${ }^{8}$ involved Si nanocrystal samples consisting of $100 \mathrm{~nm}$ silicon dioxide layers (wet thermally grown on $\mathrm{Si}$ substrates) that were implanted at room temperature with 35 $\mathrm{keV} \mathrm{Si}^{+}$ions to a fluence of $4 \times 10^{16} \mathrm{Si} / \mathrm{cm}^{2}$. The samples were annealed at $1100{ }^{\circ} \mathrm{C}$ for $10 \mathrm{~min}$ in vacuum (base pres-

\footnotetext{
a) Also at the Jet Propulsion Laboratory, Pasadena, CA 91109

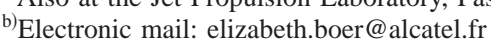

sure $<8 \times 10^{-7}$ Torr) to allow the nucleation and growth of silicon nanocrystals (size $\sim 2-6 \mathrm{~nm}$ ). The AFM used was a Park Scientific (Thermomicroscopes) Autoprobe CP, operating in noncontact mode (driving frequency above resonance) using highly doped $\mathrm{Si}$ tips (spring constant $k \sim 3 \mathrm{~N} / \mathrm{m}$ ) on triangular cantilevers from commercial sources. ${ }^{30}$ Charge was injected into the samples by applying a voltage to the AFM tip, disengaging the microscope feedback and lowering the tip toward the sample. After charge transfer the feedback was reengaged, the tip grounded, and noncontact mode images made.

The tapping mode experiments ${ }^{9}$ involved samples of isolated Si nanocrystals made by an aerosol method and deposited on a $100 \mathrm{~nm}$ thermally grown $\mathrm{SiO}_{2}$ layer on a $\mathrm{Si}$ substrate. In this case, the AFM tip was used to inject charge into isolated single nanoparticles that were subsequently imaged in the tapping mode. The microscope was a Digital Instruments Nanoscope III (driving frequency below resonance) and the tips were $\mathrm{Cr}$-coated $\mathrm{Si}$ (spring constant $k$ $\sim 2 \mathrm{~N} / \mathrm{m}$, from DI) on rectangular cantilevers.

\section{ELECTROSTATIC MODEL}

The electrostatic force between the grounded tip and charged sample was calculated using the method of images. The tip (Cr-coated or made of highly doped $\mathrm{Si}$ ) was approximated as a grounded, metallic sphere. This shape was chosen as it is an adequate approximation for our experiments (tipsample distance $d \lesssim 10 \mathrm{~nm}$ ) and is simpler than more realistic conical geometries. ${ }^{15,31}$ With tip doping $>10^{19} \mathrm{~cm}^{-3}$ and the resultant Debye length $<5 \AA$, treating the Si tip as metallic is an excellent assumption for typical tip-sample spacing in the noncontact mode. The charge on the sample was assumed to be only on the surface and the charge distribution consisted of a disk or shell upon which was imposed a grid (typical grid spacing, $5 \mathrm{~nm}$ ). The total charge was distributed uniformly over the grid points and any polarization effects and image charge in the substrate were ignored. These assumptions are justified for our particular samples, where a thick oxide separates the charge and the substrate and the charge is believed trapped on a circular area on the surface (ion implanted samples) or on the nanocrystal surface (single 


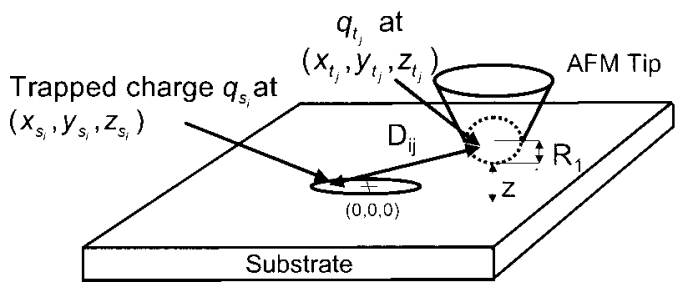

FIG. 1. Schematic for electrostatic mode model.

Si particle samples) as a result of the charge injection process. This is a first approximation-the model may be expanded to include arbitrary two- or three-dimensional charge distributions, materials effects, ${ }^{13}$ and more realistic tip shapes. The electrostatic interaction between the tip and sample was found by taking each charged grid point and finding the resultant image charge induced in the grounded tip. ${ }^{32}$ Once all the image charges (i.e., one for each grid point) were determined, the total Coulomb force was found by summing the interaction of each grid point charge with each image charge, i.e.,

$$
\mathbf{F}_{\text {electrostatic }}=\sum_{i, j} \frac{q_{s_{i}} q_{t_{j}}}{4 \pi \varepsilon_{0} D_{i j}^{2}} \hat{d}_{i j},
$$

where $q_{s_{i}}$ is the $i$ th grid point charge, $q_{t_{j}}$ is the $j$ th image charge, $D_{i j}$ the distance between them, and $\hat{d}_{i j}$ a unit vector (see Fig. 1).

\section{MODEL FOR NONCONTACT MODE IMAGING}

Most ambient atomic-force microscopes produce topographic images by exciting the AFM cantilever close to its resonant frequency and by using a feedback signal to adjust the tip-sample spacing so that the cantilever's oscillation amplitude remains constant at a set-point value. In true noncontact mode, the oscillation amplitude of the cantilever is much smaller than the tip-sample spacing, and the AFM feedback may be said to maintain a constant force gradient between tip and sample. ${ }^{21}$ For this statement to hold, the tip must never "tap" the surface (i.e., the interaction may only involve van der Waals (vdW) forces and no contact potentials) and the tip may sample only a small part of the force curve during a period of oscillation. It is also assumed in this case that the tip-surface interaction affects only the resonant frequency of the cantilever, and does not significantly influence the tip oscillation amplitude at resonance. Finally, $k$ $\geqslant F_{t-s}^{\prime}$ is another condition required, where $k$ is the spring constant of the cantilever, and $F_{t-s}^{\prime}$ is the force gradient between tip and sample, so that the force gradient is only a small perturbation to the harmonic motion of the cantilever. ${ }^{33}$
In this case, the tip-sample force $F_{t-s}$ can be expanded to first order, $F_{t-s}(z) \sim F_{t-s}\left(z=z_{L 0}\right)+F_{t-s}^{\prime}\left(z=z_{L 0}\right)\left(z-z_{L 0}\right)$ ( $z_{L 0}$ is the equilibrium position of the tip), and $F_{t-s}^{\prime}$ has the effect of changing the effective spring constant of the cantilever so that $k_{\mathrm{eff}}=k-F_{t-s}^{\prime}$, thus producing a shifted resonant frequency,

$$
\widetilde{\omega}_{0}=\sqrt{\frac{k_{\text {eff }}}{m^{*}}},
$$

where $m^{*}$ is the effective mass of the cantilever.

\section{A. Model (noncontact mode, nanoparticle ensemble)}

To produce calculated images of charge for noncontact mode imaging, we chose to solve (using Newton's method) the following equation:

$$
\left(\frac{\partial F_{z}}{\partial z}\right)_{\text {total }}=\left(\frac{\partial F_{z}}{\partial z}\right)_{\mathrm{vdW}}+\left(\frac{\partial F_{z}}{\partial z}\right)_{\text {electrostatic }}=\text { constant, }
$$

for $z$, the height of the tip above a flat plane (see Fig. 1). Note that we consider only the $z$ component of the force since in normal AFM operation only that component is sensed. We include only van der Waals and electrostatic forces and omit the negligible contribution from air damping. Here the "constant" represents the set-point force gradient which is related to the set-point amplitude in the following way. The tip amplitude as a function of frequency $\omega$ is $^{34}$

$$
A(\omega)=\frac{a_{d} \omega_{0}^{2}}{\sqrt{\left(\frac{k-F_{t-s}^{\prime}}{m^{*}}-\omega^{2}\right)^{2}+\frac{\omega_{0}^{2} \omega^{2}}{Q^{2}}}},
$$

with $A$, the measured oscillation amplitude; $a_{d}$, driving amplitude; $\omega_{0}$, resonant frequency of the cantilever; $m^{*}$, effective mass; $Q$, quality factor; $k$, spring constant; and $F_{t-s}^{\prime}$, force gradient. Assuming $a_{d}, \omega_{0}, \omega, m^{*}, Q$, and $k$ are constants, maintaining a constant cantilever amplitude may be considered equivalent to maintaining a constant force gradient. The set-point force gradient may be found experimentally by measuring the resonant frequency and the resonant frequency shift and using the approximation $\Delta \omega / \omega_{0}$ $\approx \frac{1}{2} F^{\prime} / k$ (where $\Delta \omega=\omega_{0}-\widetilde{\omega}_{0}$ ), found by the expanding expression (2) above.

The equation for the van der Waals force gradient was found by taking the derivative and $z$ component of the following expression determined by integrating the LennardJones pair potential, assuming a spherical shape for the tip and a flat plane for the sample (valid for $z \ll R$ ): ${ }^{34}$

TABLE I. Parameters for estimation of the Hamaker constant.

\begin{tabular}{cccc}
\hline \hline Symbol & Parameter & Value & Source \\
\hline$\rho_{\mathrm{Si}}$ & Number density of $\mathrm{Si}$ & $5.0 \times 10^{22} \mathrm{~cm}^{-3}$ & Ref. 35 \\
$\rho_{\mathrm{SiO}}$ & Number density of $\mathrm{SiO}_{2}$ & $6.81 \times 10^{22} \mathrm{~cm}^{-3}$ & Ref. 36 \\
$\varepsilon$ & Lennard-Jones potential parameter (for Ar) & $1.67 \times 10^{-21} \mathrm{~J}$ & Ref. 37 \\
$\sigma$ & Lennard-Jones potential parameter (for Ar) & $0.34 \mathrm{~nm}$ & Ref. 37 \\
\hline \hline
\end{tabular}




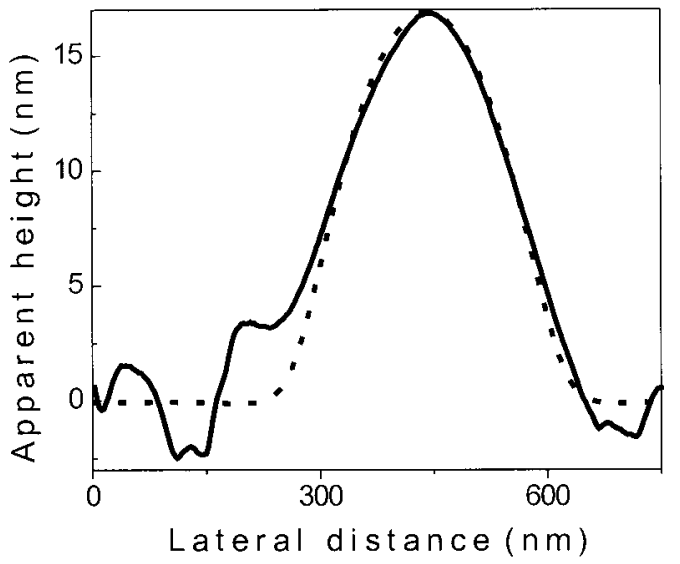

FIG. 2. Experimental image (solid line) and calculated image (dotted line) of charge deposited in a $\mathrm{SiO}_{2}$ film containing $\mathrm{Si}$ nanocrystals. From the model, the imaged charge is estimated to be $\sim 350$ electrons, over an area of radius $170 \mathrm{~nm}\left(\sim 60 \mathrm{nC} / \mathrm{cm}^{2}\right)$.

$$
\mathbf{F}_{\mathrm{vdW}}=\frac{H R}{6 z^{2}}\left(-1+\frac{\sigma^{6}}{30 z^{6}}\right) \hat{z} .
$$

The symbol $H$ represents the Hamaker constant and was estimated from $H=4 \pi^{2} \varepsilon \rho_{\mathrm{Si}_{i}} \rho_{\mathrm{SiO}_{2}} \sigma^{6} \sim 3 \times 10^{-19} \mathrm{~J}$ (symbols and values in Table $\mathrm{I}^{35-37}$ ), and $R$ is the tip radius of curvature, measured from scanning electron micrographs $(2 * R$ $\sim 105 \mathrm{~nm}$ ). The electrostatic contribution is found by taking the derivative and $z$ component of Eq. (1).

\section{B. Model results}

Figure 2 shows an experimental line scan (solid line) of a charged region of an etched $\mathrm{SiO}_{2}$ film containing silicon nanocrystals ${ }^{8}$ superimposed on a calculated AFM scan (dotted line), determined using the model described in Sec. IV A. The injected charge is imaged as a protrusion on the surface due to the extra electrostatic force interaction between the charged sample and the induced image charge in the tip. The calculated AFM scan was found by first assuming a charge distribution (i.e., total charge and charged disk radius), calculating a scan, comparing the result to the experimental data, then adjusting the charge distribution, and recalculating the result, and so on, until a best fit to the two parameters (charge and disk radius) was found. ${ }^{8}$ From the model, the imaged charge in Fig. 2 was estimated to be $\sim 350$ electrons, over an area of radius $170 \mathrm{~nm}\left(\sim 60 \mathrm{nC} / \mathrm{cm}^{2}\right)$.

\section{Charge sensitivity}

When analyzing charge storage devices and imaging small number of charges, it is important to know the sensitivity of the technique being applied. For this reason, the model of Sec. IV A was used to estimate the AFM sensitivity to charge in noncontact mode. The error in the height measurement was estimated to be $\sim 0.4-0.7 \mathrm{~nm}$ by repeated measurement of a $4 \mathrm{~nm}$ particle under regular conditions. Figure 3 shows how the minimum detectable number of electrons varies with tip-sample spacing for three different types of charge distributions: a point charge (ם), charge spread out

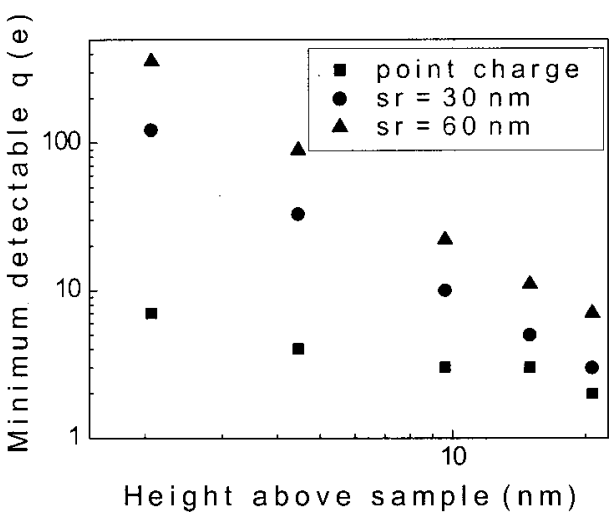

FIG. 3. Minimum detectable number of electrons as a function of the tipsample spacing (tip radius $R_{1} \sim 52 \mathrm{~nm}$, charge region radii $\mathrm{sr}=0,30$, and 60 $\mathrm{nm})$. Note the counterintuitive result that the charge sensitivity increases with increasing distance, due to the fact the van der Waals force falls off more quickly with distance than the electrostatic force.

over a disk with radius $30 \mathrm{~nm}(\mathbf{O})$ and charge over an area with a $60 \mathrm{~nm}$ radius $(\boldsymbol{\Delta})$. The nonintuitive result is that the microscope is more sensitive to charge as the tip-sample spacing increases. This can be attributed to the fact that the van der Waals force falls off more quickly with distance than the electrostatic force-thus, as the tip moves away from the sample, the relative contribution of the electrostatic force gradient to the total force gradient increases with respect to the van der Waals contribution. This curve reaches a minimum when the minimum detectable change in force gradient [estimated to be $\sim 10^{-5}-10^{-6} \mathrm{~N} / \mathrm{m}$ (Ref. 33)] due to electrostatic forces is reached at a height of $\sim 50 \mathrm{~nm}$.

Figure 4 shows the variation of the minimum detectable number of electrons as a function of tip radius. This result shows that the greatest sensitivity is found when the charge distribution is of order the same physical size as the tip-for a point charge, the greatest sensitivity of an AFM operating in noncontact mode with typical parameters and cantilevers is on the order of a few electrons. Single electron resolution in normal AFM operation would require parameter optimization.

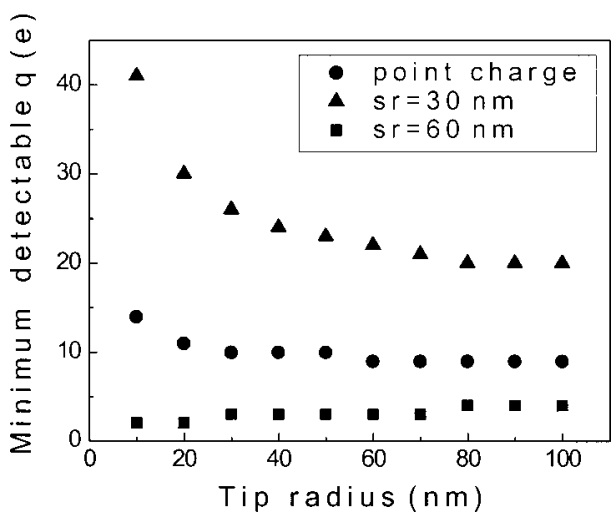

FIG. 4. Minimum detectable number of electrons as a function of tip radius. Note that the best sensitivity occurs when the tip is of the same size as the charge distribution. 
TABLE II. Parameters for the tapping mode model.

\begin{tabular}{|c|c|c|c|}
\hline Symbol & Parameter & Value & Source \\
\hline$k$ & Spring constant & $2.16 \mathrm{~N} / \mathrm{m}$ & $\begin{array}{c}k=\frac{E w t^{3}}{4 l^{3}}[\text { Ref. 34] } \\
E \text { (Young's modulus of } \mathrm{Si})=1.3 \times 10^{11} \mathrm{~N} / \mathrm{m}^{2}, \\
w(\text { width })=28 \mu \mathrm{m}, \\
t \text { (thickness) }=3 \mu \mathrm{m}, \\
l \text { (length) }=225 \mu \mathrm{m}, \text { Ref. } 39\end{array}$ \\
\hline$m^{*}$ & Effective mass & $1 \times 10^{-11} \mathrm{~kg}$ & $m^{*}=k / \omega_{0}^{2}$ or $0.24 m$, Ref. 38 \\
\hline$a_{d}$ & Driving amplitude & $0.22 \mathrm{~nm}$ & $\begin{array}{c}\text { Found by matching the calculated } \\
\text { and measured free } \\
\text { amplitudes }(32 \mathrm{~nm}) .\end{array}$ \\
\hline$\gamma$ & Dissipation coefficient & $3 \times 10^{-8} \mathrm{~kg} / \mathrm{s}$ & $\begin{array}{c}\gamma=m * \omega_{0} / Q, \omega_{0} \text { measured, } \\
Q=\omega_{0} / \Delta \omega, \Delta \omega \text { equal to } \\
\text { width } \\
\text { of resonance, i.e., width at } \\
1 / \sqrt{2} A_{\text {max }} \text { on an amplitude- } \\
\text { frequency curve. }\end{array}$ \\
\hline
\end{tabular}

\section{TAPPING MODE MODEL}

When the tip oscillation amplitude is large compared to the tip-sample spacing, the tip now experience a wide range of force values during an oscillation cycle, including a repulsive contact force when it "taps" the surface. In this case the conditions stated in Sec. IV no longer hold and a simple "noncontact" model can no longer be used. The microscope feedback still maintains a constant tip oscillation amplitude during scanning, but now the amplitude as a function of driving frequency can no longer be approximated by an analytical equations such as Eq. (4), and the amplitude at a specific tip-sample distance must be found by other means. To investigate this situation we take the cantilever to be a point mass of effective mass $m^{*}$ at the end of a massless spring ( $m^{*}=n m$, where $m$ is the mass of the cantilever, and $n$ depends on the cantilever geometry ${ }^{38}$ ). We have examined the equation for a forced harmonic oscillator:

$$
m^{*} \ddot{z}+\gamma \dot{z}+k\left(z-z_{L 0}\right)=F_{0} \cos (\omega t)+\left[F_{t-s}(z, t)\right]_{z},
$$

where $k$ is the cantilever spring constant, $m^{*}$ the effective mass, $z_{L 0}$ the equilibrium position of the cantilever, $F_{0}$ $=k a_{d}$ with $a_{d}$ the driving amplitude, $\gamma$ is the damping constant, $\omega$ is the angular driving frequency (chosen slightly below resonance, see Table $\mathrm{II}^{38,39}$ for values used), and $\left[F_{t-s}(z, t)\right]_{z}$ is the $z$ component of the tip-sample interaction force. This last term has three components: one due to the contact or tapping force interaction, one from the van der Waals force, and the third from the electrostatic force.

\section{A. Model (tapping mode, single nanoparticle)}

For this model, we considered a charged spherical particle (see Fig. 5). The electrostatic force was calculated as described in Sec. III, by taking the tip at its mean height above the sample during an oscillation and finding the Coulomb interaction between the charge on a half or full shell grid on the particle surface and the induced image charge in the grounded tip. For the van der Waals force, the interaction (a) between two spheres (the tip and particle) and (b) a sphere and plane (the tip and substrate) were added, using the full equations of Hamaker: ${ }^{40}$

(b) $\quad \mathbf{F}_{\mathrm{vdW}}=\left\{\begin{array}{c}-\frac{H}{3}\left(\frac{32 R_{1}^{3} R_{2}^{3}\left(r+\xi_{0}+R_{1}+R_{2}\right)}{\left(r+\xi_{0}\right)^{2}\left(r+\xi_{0}+2 R_{1}\right)^{2}\left(r+\xi_{0}+2 R_{2}\right)^{2}\left(r+\xi_{0}+2\left(R_{1}+R_{2}\right)\right)^{2}}\right) \hat{r}, \\ \frac{H}{24 R_{1}}\left(\frac{2}{x}-\frac{1}{x^{2}}-\frac{2}{x+1}-\frac{1}{(x+1)^{2}}\right) \hat{z}, \quad x=\frac{\left(d+\xi_{0}\right)}{2 R_{1}},\end{array}\right.$

where $d$ and $r$ are defined in Fig. 5. The parameter $\xi_{0}$ is determined by matching the value of $F_{\mathrm{vdW}}$ with the contact force at contact. Here, contact is defined as the tip-sample position at which contact mechanics must be applied. Note that the dimensions $d$ and $z$ in Fig. 5 are measured with respect to the surface of the plane when the AFM tip is not in close proximity. For large energies of adhesion, however, the plane and tip may bulge toward each other, meaning contact may occur for $d>0$.

The question of what form the contact force should take 


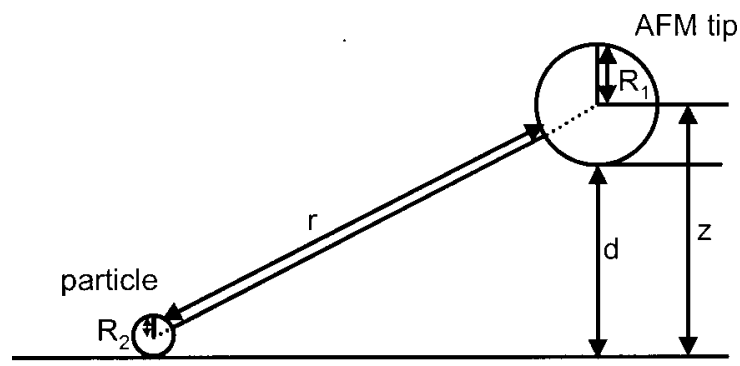

FIG. 5. Definition of symbols in tapping model equations.

is not a trivial one. ${ }^{23,41,42}$ Some possibilities are the Hertz theory $^{43}$ (includes no adhesion), the Johnson-KendallRoberts (JKR) theory (good for soft samples), ${ }^{44}$ DerjaguinMuller-Toporov (DMT) theory (best for rigid samples), ${ }^{45}$ the JKR-DMT transition formalism (appropriate for all tipsample parameters), ${ }^{46}$ Muller-Yushenko-Derjaguin/ Hughes-White (MYD/BHW) theory (a general theory), ${ }^{47,48}$ and the impact oscillator model (consisting of a harmonically driven harmonic oscillator undergoing impacts with an infinitely rigid object). ${ }^{49,50}$ In considering these theories we assume the tip and sample are elastic and ignore shear forces, ${ }^{42}$ hydrodynamic damping (small compared to the other forces involved), ${ }^{21}$ and other possible contributions to the total force such as capillary forces. Our goal here was not to perfectly model the tapping interaction, but rather to use an applicable model to interpret charge images. To this end we investigated briefly both the JKR and DMT theories.

The parameter $\mu$ first defined by Muller, Yushenko, and Derjaguin $^{47}$ may be used to delineate which of the JKR and DMT theories should be applied:

$$
\mu=\frac{32}{3 \pi}\left[\frac{2 R W^{2}}{\pi E^{* 2} z_{0}^{3}}\right]^{1 / 3} .
$$

see Tables III and IV $\mathrm{V}^{51,52}$ for symbol definitions. Roughly speaking, for $\mu \gg 1$, JKR is the better theory to choose (i.e., for soft samples, large energies of adhesion and large tip radii) and when $\mu \ll 1$, DMT is the appropriate theory (i.e., for rigid systems, low adhesion, and small radii of curvature). ${ }^{53}$ In our experiments in tapping mode, we have used a Si tip coated with chromium on Si samples covered with a native or thermally grown oxide, ${ }^{9}$ and hence, estimate a value of the adhesion energy $W\left[W=W_{12} \sim 2 \sqrt{\gamma_{1}^{d} \gamma_{2}^{d}}\right.$ (Ref. 54) from the surface energies of $\mathrm{Cr}\left[\gamma_{1}^{d} \sim 2070 \mathrm{~mJ} / \mathrm{m}^{2}\right.$ (Ref. 55) $]$ and $\mathrm{SiO}_{2}\left[\gamma_{2}^{d} \sim 70 \mathrm{~mJ} / \mathrm{m}^{2}\right.$ (Ref. 56) $]$. With the appropriate parameters for our experiments (see Tables III and IV) we get values of $\mu \sim 2$ for the flat part of our samples and $\mu \sim 1$ when imaging a $28 \mathrm{~nm}$ particle. While we are clearly in the JKR-DMT transition regime, we have chosen to look at the simpler JKR and DMT theories separately.

\section{B. JKR theory}

For the JKR theory, the relationship between the force $\left(F_{\mathrm{JKR}}\right)$ and penetration depth $(d)$ is

$$
\begin{aligned}
& a^{3}=\frac{3 R}{4 E^{*}}\left(F_{\mathrm{JKR}}+3 \pi W R+\sqrt{6 \pi W R F_{\mathrm{JKR}}+9 \pi^{2} W^{2} R^{2}}\right), \\
& d=\frac{a^{2}}{R}-\sqrt{\frac{2 \pi W a}{E^{*}}}
\end{aligned}
$$

where $a$ is the radius of the contact region. Figure 6 shows a calculated force-distance curve. Equation (7) was used when the tip and sample were not in contact and Eq. (8) when in contact (taking the $z$ component, see Tables III and IV for parameters).

Figure 7 shows how the calculated tip amplitude varies with frequency when the mean tip-sample spacing is $150 \mathrm{~nm}$ (i.e., free, solid line) and when the mean tip-sample spacing is $20 \mathrm{~nm}$ (i.e., tapping, dotted line). These curves were generated by solving Eq. (6) using MATLAB/SIMULINK (ode45, based on an explicit Runge-Kutta $(4,5)$ formula, the Dormand-Prince pair); different sets of initial conditions were chosen and the equation was allowed to evolve with time until it reached a steady state in each case. Figure 8 shows an example of the tip motion as it comes to its equilibrium amplitude. Note that in Fig. 7, more than one amplitude at a specific frequency is possible in the curve generated at a tip-sample height less than the free amplitude $(\bullet)$ whether the system settled into one steady state or another depended on what initial conditions were chosen. This result for a tapping amplitude-frequency curve is qualitatively

TABLE III. Constant and material parameters for contact interaction I.

\begin{tabular}{ccc}
\hline \hline Symbol & Parameter & Value \\
\hline \hline$R_{1}$ & Tip radius of curvature & $50 \mathrm{~nm}$ \\
$R_{2}$ & Sample radius of curvature & $\left\{\begin{array}{c}14 \mathrm{~nm} \text { (particle) } \\
\infty \text { (plane) }\end{array}\right.$ \\
$R$ & Reduced radius of curvature, $1 / R=1 / R_{1}+1 / R_{2}$ & $\left\{\begin{array}{c}11 \mathrm{~nm} \text { (particle) } \\
14 \mathrm{~nm} \text { (plane) } \\
W \\
z_{0}\end{array} \quad\right.$ Work of adhesion \\
$H$ & Equilibrium interatomic distance & $0.756 \mathrm{~J} / \mathrm{m}^{2}$ \\
$E^{*}$ & Hamaker constant & $0.34 \mathrm{~nm}$ \\
$1.8 \times 10^{-18} \mathrm{~J}$ \\
\hline \hline
\end{tabular}


TABLE IV. Constants and material parameters for contact force II.

\begin{tabular}{|c|c|c|c|c|}
\hline Symbol & Parameter & Material & Value & Source \\
\hline$E_{t}$ & Young's modulus of tip & $\mathrm{Cr}$ & $27.9 \times 10^{10} \mathrm{~N} / \mathrm{m}^{2}$ & Ref. 51 \\
\hline$E_{s}$ & Young's modulus of sample & $\mathrm{SiO}_{2}$ & $7 \times 10^{10} \mathrm{~N} / \mathrm{m}^{2}$ & Ref. 52 \\
\hline$\nu_{t}$ & Poisson's ratio for tip & $\mathrm{Cr}$ & 0.21 & Ref. 51 \\
\hline$\nu_{s}$ & Poisson's ratio for sample & $\mathrm{SiO}_{2}$ & 0.17 & Ref. 52 \\
\hline
\end{tabular}

similar to experimental and computational results seen by others, ${ }^{24-28,57-59}$ with its truncated, extended peak. However, differences include curvature to the "flat" part of the truncated peak, the width of the plateau, and the small region just below resonance where, in principle, three solutions are possible.

Figure 9 shows a calculated amplitude-distance curve above a $28 \mathrm{~nm}$ particle (cantilever driven below resonance). The tip was brought in slowly from far away, and the tip motion was allowed to evolve for 1.2-3.6 ms at tip-sample spacing $d$, before moving to $d+\Delta d(\Delta d=0.1 \mathrm{~nm}$, typically). Far from the sample, the tip oscillation amplitude is constant, then is seen to increase slightly before decreasing linearly with tip-sample spacing. The initial increase in amplitude is due to the fact that the resonance frequency $\widetilde{\omega}_{0}$ is shifting down toward the cantilever driving frequency as the tip interacts with the attractive force of the sample. Occasionally in these amplitude-distance curves, discontinuous jumps to another solution were seen, especially when the tip-sample spacing was small, or when the amplitude-distance curve was generated above a particle (i.e., weaker tip-sample forces). The existence of the discontinuity also depended on $\Delta d$ [see Fig. 9(a)], suggesting more of a dependence on initial conditions ${ }^{58}$ than on the strength or other property of the attractive force. ${ }^{60}$ The features we find are similar to those seen by others experimentally and computationally, ${ }^{57,61}$ and are qualitatively similar to our experimental traces. Unlike Anczykowski, Krüger, and Fuchs, ${ }^{61}$ we calculate a step-like discontinuity for a driving frequency less than the resonant frequency.

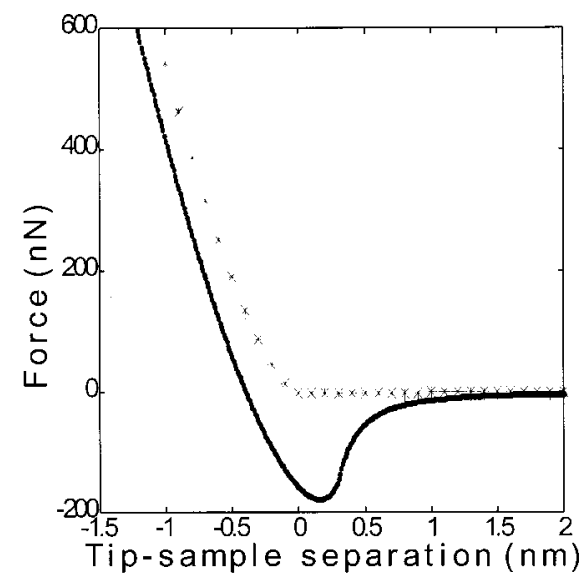

FIG. 6. Force-distance curves for a $50 \mathrm{~nm}$ radius $\mathrm{Cr}$-coated tip and a $\mathrm{SiO}_{2}$ sample, using the JKR theory for the contact force with $W=756 \mathrm{~mJ} / \mathrm{m}^{2}($ or the DMT theory with $W=10 \mathrm{~mJ} / \mathrm{m}^{2}(*)$.

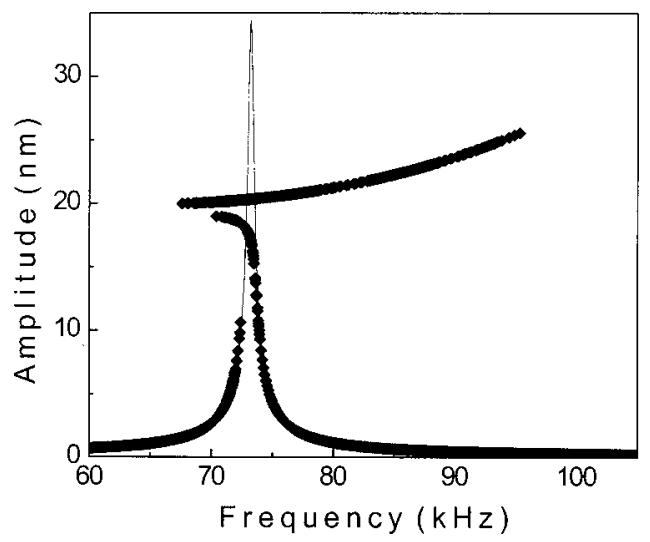

FIG. 7. Amplitude vs frequency curves: solid line, mean tip-sample spacing $150 \mathrm{~nm},(\diamond)$ mean tip-sample spacing $20 \mathrm{~nm}$. Note that when tapping more than one amplitude at the same frequency is possible.

If instead of slowly lowering the tip and allowing its motion to evolve, various initial conditions (position and velocity) are chosen for the tip at each height $d$, we generate an amplitude-distance curve, as seen in Fig. 10. Here, we have two tip-sample separations possible for one amplitude- the particular solution is again determined by which initial conditions are chosen. The solution dependence on the initial position of the tip $\left(z_{i}\right)$ with initial velocity zero was briefly investigated. It was found that when the tip was "plucked" with large displacement (i.e., $z_{i}$ larger than the maximum $z$ at steady-state amplitude), two solutions were possible, but when $z_{\text {steady state }}^{\text {min }}<z_{i}<z_{\text {steady state }}^{\max }$, in general, only one solution was found though occasionally two were seen (Fig. 11). Thus, there exist sets of initial conditions (or points in phase space) that lead to particular solutions. ${ }^{58,62}$ This phenomenon has been used to explain distortion in experimental images $57,58,62,63$ and the switch from one solution to the other has been attributed to a transition from a purely attractive to a partly repulsive interaction regime. $57,61,64$

To produce a calculated tapping mode image and ensure we arrive at an appropriate solution, at each lateral position we produce an amplitude-distance curve (such as Fig. 9) and find the tip-plane separation for the set-point amplitude. It was found that the image of a $28 \mathrm{~nm}$ particle calculated in

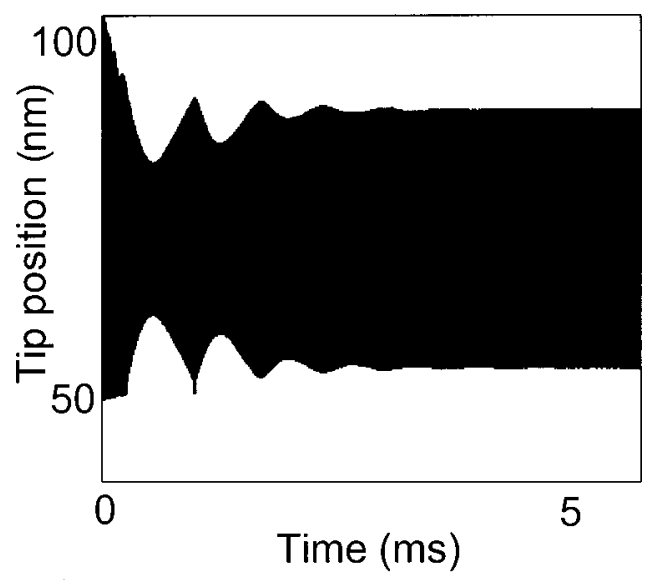

FIG. 8. Tip motion as a function of time as it evolves to steady state. 


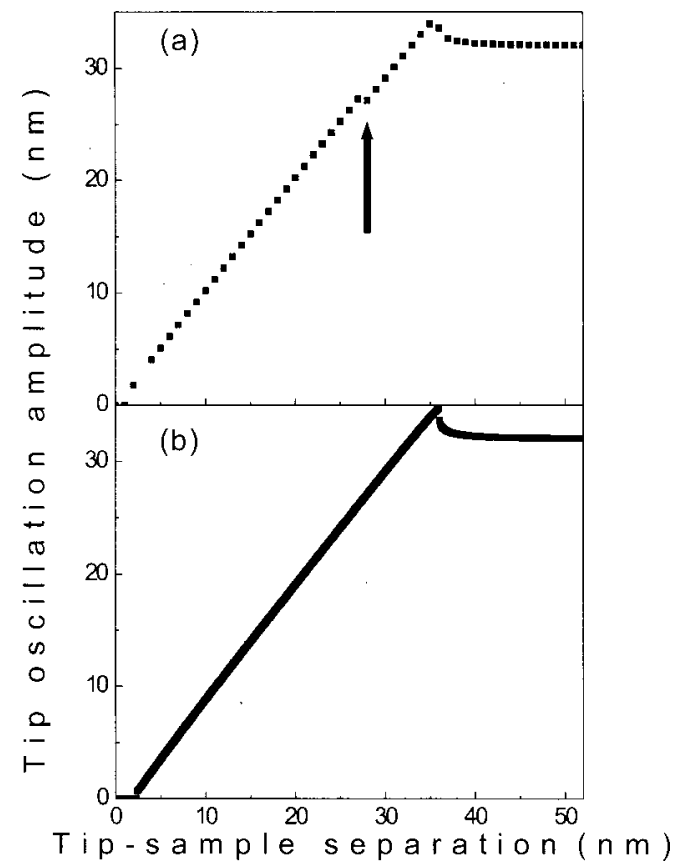

FIG. 9. Amplitude-distance curves above a $28 \mathrm{~nm}$ particle. In (a) the amplitude was calculated at $1 \mathrm{~nm}$ intervals while lowering the tip toward the surface and in (b) the amplitude was calculated every $0.1 \mathrm{~nm}$. Note the discontinuous jump in (a).

this way produced a particle height that was slightly depressed. The error increased with decreasing particle size and with increasing energy of adhesion $(W)$ - the latter suggests structures of different materials of the same height may produce slightly different AFM results. This depression in height was attributed to the fact that both the van der Waals and contact forces between a tip and nanoscale particle are smaller than those between a tip and plane, due to the large difference in the radius of curvature $\left(R_{2}\right)$ of the imaged object. To correct for this phenomenon, we used a slightly larger particle in the model to arrive at the correct measured

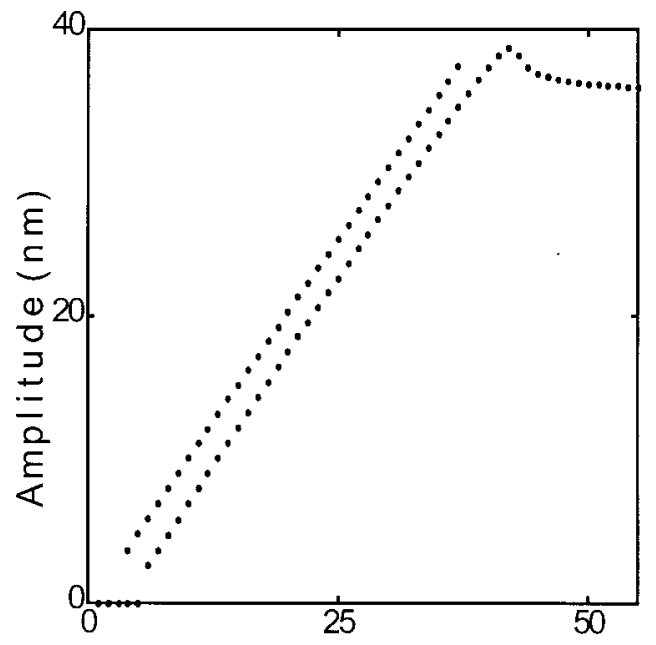

Tip-sample separation ( $\mathrm{nm})$

FIG. 10. Amplitude-distance curve showing the two possible solutions at each tip-sample distance. These curves were generated by choosing different initial conditions.

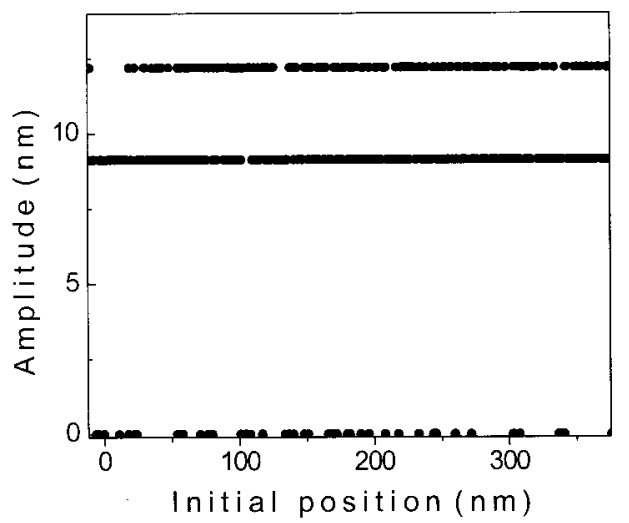

FIG. 11. Tip oscillation amplitude as a function of initial displacement of tip about its equilibrium position (initial velocity $=0$ ). Note that, in general, both solutions are possible, except for a small range about the equilibrium value where the lower amplitude solution dominates. For some initial conditions, trapping by the sample (i.e., amplitude $\sim 0$ ) was also found.

particle height (e.g., $R_{2}=14.99 \mathrm{~nm}$ instead of $14.03 \mathrm{~nm}$ ). Height anomalies have been observed experimentally in tapping mode and have been attributed to differences in adhesion (capillary forces). ${ }^{65}$

Figure 12(a) shows an experimental data series of a 28 $\mathrm{nm}$ particle that has been injected with charge with a $\mathrm{Cr}$ coated Si AFM tip ${ }^{8}$ - from the initial increase and following decrease in apparent height we conclude that charge is indeed being injected and is then dissipating. Figure 12(b) shows the calculated fits to the data in (a), determined from

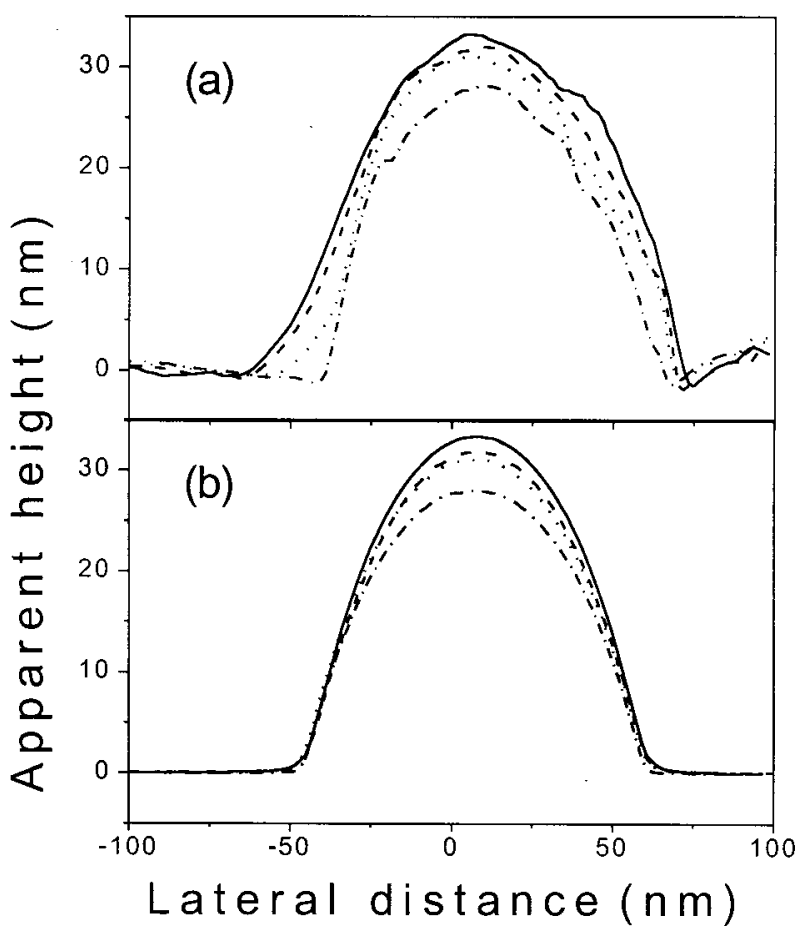

FIG. 12. (a) Experimental data and (b) fits to this data using the tapping mode model. The data represent a $28 \mathrm{~nm}$ particle before being charged (bottom trace) then 45, 217, and $527 \mathrm{~s}$ after charging (from top to bottom). In (b), calculated profiles are shown for (from bottom) $0 \mathrm{e}, 44 \mathrm{e}, 49 \mathrm{e}$, and 56e charges on the particle using the JKR theory for the contact interaction. 
TABLE V. Comparison of results for the number of charges imaged for different contact forces.

\begin{tabular}{ccc}
\hline \hline Contact theory & $W$ & Number of electrons imaged \\
\hline JKR & $756 \mathrm{~mJ} / \mathrm{m}^{2}$ & $56 \mathrm{e}, 49 \mathrm{e}, 44 \mathrm{e}$ \\
DMT & $1 \mathrm{~mJ} / \mathrm{m}^{2}$ & $49 \mathrm{e}, 43 \mathrm{e}, 38 \mathrm{e}$ \\
DMT & $10 \mathrm{~mJ} / \mathrm{m}^{2}$ & $50 \mathrm{e}, 43 \mathrm{e}, 38 \mathrm{e}$ \\
DMT/JKR & $756 \mathrm{~mJ} / \mathrm{m}^{2}$ & $56 \mathrm{e}, 48 \mathrm{e}, 43 \mathrm{e}$ \\
\hline \hline
\end{tabular}

the tapping mode model. From these fits we estimate that the initial amount of charge injected was on the order of 60 electrons.

\section{DMT theory}

Since the system is in the JKR-DMT transition regime, and since the adhesion energy $W$ is a difficult parameter to measure and might well be lower than our estimate in Sec. $\mathrm{VA}$, we have also briefly investigated tapping using the DMT theory. Here, the important equations are

$$
\begin{aligned}
& a^{3}=\frac{3 R}{4 E^{*}}\left(F_{\mathrm{DMT}}+2 \pi W R\right), \\
& d=\frac{a^{2}}{R} .
\end{aligned}
$$

Figure 6 includes a force-distance curve for this theory. We have considered adhesion energies of $W=1$ and 10 $\mathrm{mJ} / \mathrm{m}^{2}$. For the smaller $W$, we found all particle heights were imaged correctly in the calculation. For the $W=10 \mathrm{~mJ} / \mathrm{m}^{2}$ case, a slightly larger particle height $\left(R_{2}=14.49 \mathrm{~nm}\right)$ was again used to get the correct measured value. Finally, the situation $W=756 \mathrm{~mJ} / \mathrm{m}^{2}$, with a DMT interaction between the tip and particle, and a JKR interaction between the tip and plane was considered. In all cases, fits to the data in Fig. 12(a) were made and the results are summarized in Table V. The small spread in the results $(\sim 13 \%)$ shows that the exact form of the contact force is unimportant for quantitative charge imaging. Closer to the detection limit, the different force types yield a similar variation $(\sim 12 \%)$.

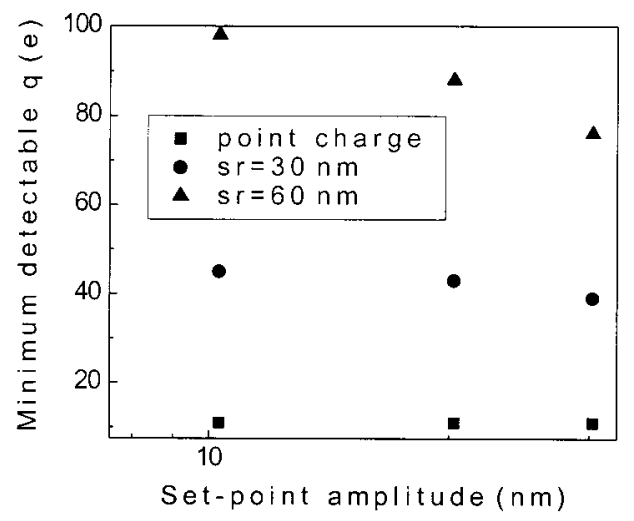

FIG. 13. Minimum detectable charge as a function of set-point amplitude, or equivalently, tip-sample spacing (tip radius $R_{1} \sim 50 \mathrm{~nm}$, charge region radii $\mathrm{sr}=0,30$, and $60 \mathrm{~nm}$ ). Analogously to the noncontact case, sensitivity increases as tip-sample spacing increases.

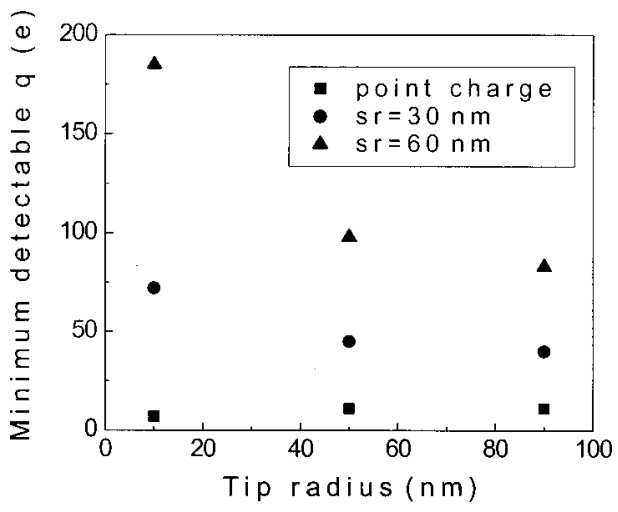

FIG. 14. Minimum detectable charge as a function of tip radius. Analogous to the noncontact case, the best sensitivity occurs when the tip and charge distribution are of the same order.

\section{Charge sensitivity}

Charge sensitivity was briefly investigated in tapping mode analogously to the noncontact mode case (Sec. IV C). Figures 13 and 14 are the corresponding graphs to Fig. 3 and 4 , respectively. Similar trends are seen in both cases. In order to achieve greatest charge sensitivity, the appropriate tip size for the charge distribution should be chosen, and the tipsample distance or set point should be optimized.

\section{E. Lateral resolution}

Besides charge sensitivity, we used the models to estimate lateral charge resolution. To do this we assumed a charge distribution consisting of two point charges (50 electrons each) arranged symmetrically on either side of the origin on the $y$ axis. We then successively reduced the distance between the two point charges and calculated an AFM scan in each case $\left(R_{1}=50 \mathrm{~nm}\right)$. These results are found in Fig. 15. From this we estimate that such point charge distributions are resolvable if they are $\sim 40-50 \mathrm{~nm}$ apart (50 nm tip radius).

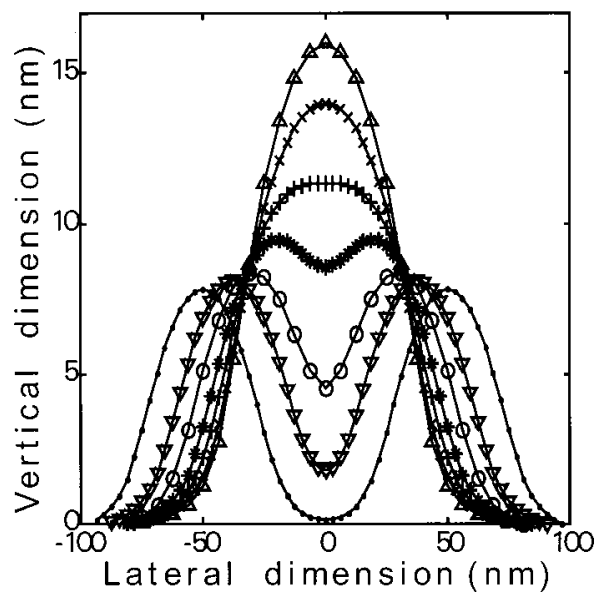

FIG. 15. Calculated AFM scans of two point charges, separated by various lateral distances. Each point charge consists of 50 electrons. In the scans shown, they are $15(\triangle), 25(\times), 35(+), 45(*), 60(\bigcirc), 75(\nabla)$, and $100(\bigcirc)$ $\mathrm{nm}$ apart. 


\section{CONCLUSIONS}

We have presented two simple models for quantitative AFM charge imaging - one valid in noncontact mode, the other in tapping mode. Comparisons among different theories for the contact force show that the exact form of this force is unimportant for quantitative charge imaging. We have applied these models to two experimental situations, have found good fits to the data, and have made quantitative estimates of the imaged charge. Estimates of the sensitivity of AFM charge imaging suggest that the charge resolution is a few electrons for standard AFM cantilevers and imaging parameters. From the models we have determined that the charge sensitivity may be improved by increasing tipsample height and choosing an appropriate tip radius for the charge distribution imaged (see, also, Refs. 60-65).

\section{ACKNOWLEDGMENTS}

The research described in this article was jointly sponsored by the National Aeronautics and Space Administration (NASA) and the Jet Propulsion Laboratory Director's Research and Development Fund, and by the National Science Foundation under Grant No. DMR98-71850.

${ }^{1}$ G. Binnig, C. Quate, and C. Gerber, Phys. Rev. Lett. 56, 930 (1986).

${ }^{2}$ C. Schonenberger and S. F. Alvarado, Phys. Rev. Lett. 65, 3162 (1990).

${ }^{3}$ B. D. Terris, J. E. Stern, D. Rugar, and H. J. Mamin, Phys. Rev. Lett. 63, 2670 (1989)

${ }^{4}$ H. Hieda, K. Tanaka, and N. Gemma, J. Vac. Sci. Technol. B 14, 1234 (1996).

${ }^{5}$ S. Morita, Y. Fukano, T. Uchihashi, T. Okusako, Y. Sugawara, Y. Yamanishi, and T. Oasa, Jpn. J. Appl. Phys., Part 2 32, L1701 (1993).

${ }^{6}$ D. M. Schaadt, E. T. Yu, S. Sankar, and A. E. Berkowitz, Appl. Phys. Lett. 74, 472 (1999)

${ }^{7}$ J. T. Jones, P. M. Bridger, O. J. Marsh, and T. McGill, Appl. Phys. Lett. 75, 1326 (1999)

${ }^{8}$ E. A. Boer, L. D. Bell, M. L. Brongersma, H. A. Atwater, and R. C. Flagan, Appl. Phys. Lett.

${ }^{9}$ E. A. Boer, L. D. Bell, M. L. Brongersma, H. A. Atwater, M. L. Ostrant, and R. C. Flagan, Appl. Phys. Lett. 78, 3133 (2001).

${ }^{10}$ Y. Martin, D. W. Abraham, and H. K. Wickramasinghe, Appl. Phys. Lett. 52, 1103 (1988).

${ }^{11}$ J. E. Stern, B. D. Terris, H. J. Mamin, and D. Rugar, Appl. Phys. Lett. 53, 2717 (1988).

${ }^{12}$ D. M. Taylor, Thin Solid Films 331, 1 (1998).

${ }^{13}$ N. A. Burnham, R. J. Colton, and H. M. Pollock, Phys. Rev. Lett. 69, 144 (1992).

${ }^{14}$ S. Gäomez-Moñivas and J. J. Sáenz, Appl. Phys. Lett. 76, 2955 (2000).

${ }^{15}$ S. Belaidi, P. Girard, and G. Leveque, J. Appl. Phys. 81, 1023 (1997).

${ }^{16}$ X. de la Broïse, M. Lannoo, and C. Delerue, J. Appl. Phys. 82, 5589 (1997).

${ }^{17}$ S. Watanabe, K. Hane, T. Ohye, M. Ito, and T. Goto, J. Vac. Sci. Technol. B 11, 1774 (1993).

${ }^{18}$ Z.-Y. Li, B.-Y. Gu, and G.-Z. Yang, Phys. Rev. B 57, 9225 (1998).

${ }^{19}$ P. J. S. Baird, J. R. Bowler, and G. C. Stevens, in Inst. Phys. Conf. Ser. 163, 381 (1999).

${ }^{20}$ R. Nishi, N. Yoshizumi, T. Outa, Y. Sugawara, S. Morita, and T. Okada, Jpn. J. Appl. Phys., Part 2 37, L417 (1998).

${ }^{21}$ G. Y. Chen, R. J. Warmack, A. Huang, and T. Thundat, J. Appl. Phys. 78, 1465 (1995).

${ }^{22}$ J. Chen, R. K. Workman, D. Sarid, and R. Höper, Nanotechnology 5, 199 (1994).

${ }^{23}$ N. A. Burnham, O. P. Behrend, F. Oulevey, G. Gremaud, P. J. Gallo, D.
Gourdon, E. Dupas, A. S. Kulik, H. M. Pollock, and G. A. D. Briggs, Nanotechnology 6, 67 (1997).

${ }^{24}$ J. P. Spatz, S. Sheiko, M. Möller, A. G. Winkler, and P. Reineker, Nanotechnology 6, 40 (1995).

${ }^{25}$ J. Tamayo and R. Garcia, Langmuir 12, 4430 (1996).

${ }^{26}$ M. Hoummady, E. Rochat, and E. Farnault, Appl. Phys. A: Mater. Sci. Process. A66, S935 (1998).

${ }^{27}$ G. Bar, R. Brandsch, and M.-H. Whangbo, Surf. Sci. 411, L802 (1998).

${ }^{28}$ L. Wang, Appl. Phys. Lett. 73, 3781 (1998).

${ }^{29}$ L. Nony, R. Boisgard, and J. Aimé, J. Chem. Phys. 111, 1615 (1999).

${ }^{30}$ Thermomicroscopes, formally Park Scientific.

${ }^{31}$ S. Belaidi, P. Girard, and G. Leveque, Microelectron. Reliab. 37, 1627 (1997).

${ }^{32}$ J. Jackson, Classical Electrodynamics, 2nd ed. (Wiley, New York, 1975).

${ }^{33}$ Y. Martin, C. C. Williams, and H. K. Wickramasinghe, J. Appl. Phys. 61, 4723 (1987).

${ }^{34}$ D. Sarid, Scanning Force Microscopy (Oxford University Press, New York, 1994).

${ }^{35}$ B. G. Streetman, Solid State Electronic Devices, 3rd ed. (Prentice Hall, Englewood Cliffs, NJ, 1990).

${ }^{36}$ K. N. Tu, J. W. Mayer, and L. C. Feldman, Electronic Thin Film Science (Macmillan, New York, 1992).

${ }^{37}$ C. Kittel, Introduction to Solid State Physics, 7th ed. (Wiley, New York, 1996).

${ }^{38}$ G. Y. Chen, R. J. Warmack, T. Thundat, D. P. Allison, and A. Huang, Rev. Sci. Instrum. 65, 2532 (1994).

${ }^{39}$ Tip dimensions provided by distributor (Digitial Instruments).

${ }^{40}$ H. Hamaker, Physica IV, 1058 (1937).

${ }^{41}$ D. S. Rimai, D. J. Quesnel, and A. A. Busnaina, Colloids Surf., A 165, 3 (2000).

${ }^{42}$ W. Unertl, J. Vac. Sci. Technol. A 17, 1779 (1999).

${ }^{43}$ H. Hertz, J. Reine Angew. Math. 92, 156 (1881).

${ }^{44}$ K. L. Johnson, K. Kendall, and A. D. Roberts, Proc. R. Soc. London, Ser. A 324, 301 (1971).

${ }^{45}$ B. V. Derjaguin, V. M. Muller, and Y. P. Toporov, J. Colloid Interface Sci. 53, 314 (1975).

${ }^{46}$ D. Maugis, Contact, Adhesion and Rupture of Elastic Solids (Springer, Berlin, 2000).

${ }^{47}$ V. M. Muller, V. S. Yushenko, and B. V. Derjaguin, J. Colloid Interface Sci. 77, 91 (1980).

${ }^{48}$ B. D. Hughes and L. R. White, J. Mech. Appl. Math. 32, 445 (1979).

${ }^{49}$ J. Berg and G. A. D. Briggs, Phys. Rev. B 55, 14899 (1997).

${ }^{50}$ M. V. Salapaka, D. J. Chen, and J. P. Cleveland, Phys. Rev. B 61, 1106 (2000).

${ }^{51}$ Smithells Metals Reference Book, 7th ed., edited by E. A. Brandes and G. B. Brook (Reed Educational and Professional, Oxford, 1998).

${ }^{52}$ Properties of Silicon (INSPEC, London, 1988).

${ }^{53}$ V. M. Muller, B. V. Derjaguin, and Y. P. Toporov, Colloids Surf. 7, 251 (1983).

${ }^{54}$ J. Israelachvili, Intermolecular and Surface Forces, 2nd ed. (Academic, London, 1992).

${ }^{55}$ V. K. Kumikov and K. B. Bhokonov, J. Appl. Phys. 54, 1346 (1983).

${ }^{56}$ E. Papirer and H. Balard, The Surface Properties of Silicas (Wiley, New York, 1998).

${ }^{57}$ A. Kühle, A. H. Sørensen, and J. Bohr, J. Appl. Phys. 81, 6562 (1997).

${ }^{58}$ M. Marth, D. Maier, J. Honerkamp, R. Brandsch, and G. Bar, J. Appl. Phys. 85, 7030 (1999).

${ }^{59}$ P. Gleyzes, P. K. Kuo, and A. C. Boccara, Appl. Phys. Lett. 58, 2989 (1991).

${ }^{60}$ B. Anczykowski, J. P. Cleveland, D. Krüger, V. Elings, and H. Fuchs, Appl. Phys. A: Mater. Sci. Process. A66, S885 (1998).

${ }^{61}$ B. Anczykowski, D. Krüger, and H. Fuchs, Phys. Rev. B 53, 15485 (1996).

${ }^{62}$ R. Garcia and A. San Paulo, Phys. Rev. B 61, R13381 (2000).

${ }^{63}$ A. Kühle, A. H. Sørensen, H. B. Zandbergen, and J. Bohr, Appl. Phys. A: Mater. Sci. Process. A66, S329 (1998).

${ }^{64}$ R. Garcia and A. San Paulo, Ultramicroscopy 82, 79 (2000).

${ }^{65}$ S. J. T. Van Noort, K. O. Van der Werf, B. G. De Grooth, N. F. Van Hulst, and J. Greve, Ultramicroscopy 69, 117 (1997). 